\title{
A Comprehensive, Multi-Modal Strategy to Mitigate Alzheimer's Disease Risk Factors Improves Aspects of Metabolism and Offsets Cognitive Decline in Individuals with Cognitive Impairment
}

Ginger Schechter ${ }^{\mathrm{a}}$, Gajendra Kumar Azad ${ }^{\mathrm{b}, \mathrm{c}}$, Rammohan Rao ${ }^{\mathrm{a}, \mathrm{d}}$, Allison McKeany ${ }^{\mathrm{a}}$, Matthew Matulaitis ${ }^{\mathrm{a}}$, Denise M. Kalos ${ }^{\mathrm{a}}$ and Brian K. Kennedy,d,e,f,g,*

${ }^{a}$ Affirmativ Health, Sonoma, CA, USA

${ }^{\mathrm{b}}$ Department of Biochemistry, Yong Loo Lin School of Medicine, National University of Singapore, Singapore ${ }^{\mathrm{c}}$ Department of Zoology, Patna University, Patna, Bihar, India

${ }^{\mathrm{d}}$ Buck Institute for Research on Aging, Novato, CA, USA

${ }^{\mathrm{e}}$ Department of Physiology, Yong Loo Lin School of Medicine, National University of Singapore, Singapore

${ }^{\mathrm{f}}$ Centre for Healthy Longevity, National University Health System, Singapore

${ }^{\mathrm{g}}$ Singapore Institute of Clinical Sciences, $A *$ STAR, Singapore

\begin{abstract}
.
Background: Alzheimer's disease (AD) is a chronic condition that progresses over time. While several therapeutic approaches have been developed, none have substantially altered disease progression. One explanation is that the disease is multi factorial. Objective: Using the Affirmativ Health Personal Therapeutic Program (PTPr), we sought to determine whether a comprehensive and personalized program could improve cognitive and metabolic function in individuals diagnosed with subjective cognitive impairment, mild cognitive impairment, and early stage AD.

Methods: 35 individuals submitted blood samples and Montreal Cognitive Assessment (MoCA) scores, and answered intake questions. Individuals and caregivers participated in a four-day immersion program, which included Personal Therapeutic Plans (PTP), consultations with clinical practitioners, and explanations of the PTPr and PTP. Participants had follow-up by telemonitoring, with repeat blood sample analysis, updates regarding lifestyle choices, current medications and supplements, and MoCA testing at least once between 3 and 12 months after the PTPr.

Results: By comparing baseline to follow-up testing, we determined several risk factor scores, including blood glucose and insulin levels, and levels of vitamins B12, D3, and E, improved either in the entire participant pool or specifically in
\end{abstract}

\footnotetext{
${ }^{*}$ Correspondence to: Brian K. Kennedy, Department of Biochemistry, Yong Loo Lin School of Medicine, National University of Singapore, Singapore, 117596. E-mail: bkennedy@ nus.edu.sg.
} 
individuals with measures outside the normal range for specific factors. MoCA scores were stabilized in the entire participant pool and significantly improved in individuals scoring 24 or less at baseline.

Conclusion: Our findings provide evidence that a comprehensive and personalized approach designed to mitigate AD risk factors can improve risk factor scores and stabilize cognitive function, warranting more extensive and placebo-controlled clinical studies.

Keywords: Alzheimer's disease, metabolism, mild cognitive impairment, personalized treatment, subjective cognitive impairment

\section{INTRODUCTION}

Despite numerous attempts and a vast commitment of resources, efforts to reverse or even blunt the progression of Alzheimer's disease (AD) have been marginally successful at best $[1,2]$. This has been attributed to many potential reasons. For instance, it has been argued that most clinical trials have been performed on individuals in relatively late stage disease progression, where the pathology may have progressed to an irreversible state [3]. Another line of thought suggests that the primary targets of therapeutic development, amyloid- $\beta$ plaques and tau tangles, may not be a major drivers of disease, or at least that their therapeutic clearance is insufficient to blunt disease progression [4, 5]. Instead, amyloid$\beta$ oligomers may be the primary culprit or, even more concerning, the role of amyloid plaques in disease progression may be overestimated [6]. Indeed, the role of other factors, such as chronic inflammation and altered metabolism, have become evident $[7,8]$. How these pathways are linked to plaques and tangles remains to be fully elucidated. Better diagnostic procedures are also indicating that age-associated dementias are more varied than expected [9]; therefore, selection of participants in previous clinical trials may not have been performed with enough specificity.

Under-represented in therapeutic approaches to $\mathrm{AD}$ are the role of risk factors and the idea that mitigation of risk factors in early stage disease may delay clinical progression [10-14]. Most studies to date have examined single interventions to target one risk factor, for instance low vitamin D3 levels [15], with little or no efficacy. Yet, there are as many genetic and lifestyle risk factors associated with $\mathrm{AD}$ and each individual is only compromised in a subset [16]. Therefore, changing one risk factor may only produce marginal outcomes and studies are beginning to support this concept [17-20].

We have tested a more holistic concept based on the hypothesis that: 1) risk factors for $\mathrm{AD}$ contribute to disease progression, 2) affected individuals have unique combinations of risk factors; and 3) that risk factors act in an additive or synergistic manner to promote disease progression. Therefore, a personalized strategy to identify and attenuate the risk factors specific to each affected individual will have a significant impact on disease progression, as has been shown with related approaches [21, 22]. Here we present pilot data testing of that concept and make the case for expansion of this approach to people in the early stages of $\mathrm{AD}$ progression.

\section{METHODS}

\section{Study design and participants}

Participants were recruited through a marketing campaign that included online digital advertising, radio programming, and participant referrals. Upon contacting the program, individuals were screened using an intake process, which included baseline Montreal Cognitive Assessment (MoCA) testing, and a questionnaire that included demographics, current medical care, past medical and surgical history, current medical history, tobacco, alcohol and recreational drug usage, current medications and supplement usage, family history, social history, current living environment, AD symptoms, mental health history, dietary patterns, allergies and anthropometric data.

After performing the above intake, participants meeting our study profile underwent genetic and blood testing for a range of parameters associated with the onset of $\mathrm{AD}$ (Table 1). Data was then processed with our proprietary software technology producing the Personalized Therapeutic Plan (PTP), which identifies the potential under-pinning causes of memory loss. Data was then screened by a physician and registered dietician to identify medical diseases or indicators that may affect memory unrelated to AD. In some cases, new clinical conditions were identified that required medical attention. Qualified candidates 
Table 1

Blood panel components

\begin{tabular}{lll}
\hline Blood Pressure & Copper, serum & Thyroid Stimulating Hormone (TSH) \\
\hline White Blood Cells (WBC) & Zinc, serum & Free T3 (fT3) \\
Red Blood Cells (RBC) & Selenium, serum & Testoterone, total \\
Hemoglobin (Hgb) & Total Cholesterol & Testosterone, free \\
Hematocrit (Hct) & HDL Cholesterol & Cortisol, AM \\
Mean Corpuscular Volume (MCV) & LDL Cholesterol & \\
Mean Corpuscular Hemoglobin (MCH) & HDL:Total Chol & \\
Platelet Count & TG:HDL & \\
Fasting Glucose, Serum & Triglycerides & \\
Fasting Insulin & Homocysteine, serum & \\
Hemoglobin A1c (HgA1c) & C-reactive Protein, high sensitivity (hs-CRP) & \\
Creatinine, Fasting & Vitamin B12, serum & \\
Glomerular Filtration Rate, estimated (eGFR) & Vitamin D, serum & \\
Magnesium, RBC & Vitamin E, serum & \\
Calcium, serum & Folate, serum & \\
\hline
\end{tabular}

were invited to participate in the Affirmativ Health Personal Therapeutic Program (PTPr).

\section{Program}

The PTPr included the above preliminary testing, the PTP and a 4-day in-person, immersion retreat, ongoing coaching, and follow-up testing. At the retreat, participants receive personalized interactions with a range of specialists, including a physician, dietitian, physical therapist, geriatric psychologist, and others. In addition, participants are coached in healthy lifestyle choices, including cooking, stress management, sleep, exercise programs, and an introduction to brain stimulation activities. Most importantly, participants are taken through a thorough review of their PTP, which includes blood panel, genetic and cognitive testing results, individual risk factors, and optimal blood panel target ranges (supported by previously published research) to achieve cognitive stabilization and/or improvement. They are also provided with strategies to mitigate those risks. As previously described, strategies include personalized dietary and exercise recommendations, stress reduction, and supplements when necessary and feasible. All recommended supplement interventions are widely used and have strong safety profiles. No prescription medications were recommended as part of treatment. At the end of the four-day window, participants are provided with a physician's version of the PTP to discuss with their primary care physicians, who are responsible for any specific clinical recommendations included in the PTP that require follow-up action.

After completing the 4-day retreat, monthly follow-up coaching calls are made to assist partici- pants with adherence to the program and to capture adherence data. Follow-up testing was performed at three or six months based on the time it took the participant to be fully adherent. Occasionally, later testing was performed.

\section{Statistical analysis}

To visualize the datasets of various parameters from human intervention samples, we used the representation methods 'line graph' and 'boxplot'. We generated the box plot for each group of datasets, as shown in the figures. The standard deviation and error bars were also calculated and added in each box plot.

The statistical analyses were carried out using Graph Pad Prism software. The statistical significance was determined by two tailed unpaired Student's $t$-test. The data were expressed as means \pm standard deviation (SD) and the $p$-value less than 0.05 were considered significant. The correlation plot between pre and post MoCA values was also generated, as shown in Fig. 1. The correlation value (R2) was calculated and the data points showed a positive linear association (R2-0.87).

\section{RESULTS}

In total, 35 people were enrolled in the program, completing initial testing, the four-day retreat, and at least one follow-up blood panel and cognitive test to allow for statistical analysis of efficacy. Participants were requested to comply with a robust program that involves several lifestyle changes and supplements. We generated feedback on adherence, finding a mean adherence score of $69.67 \%$ with a median score of $80 \%$. Statistics on the participant pool are shown in 

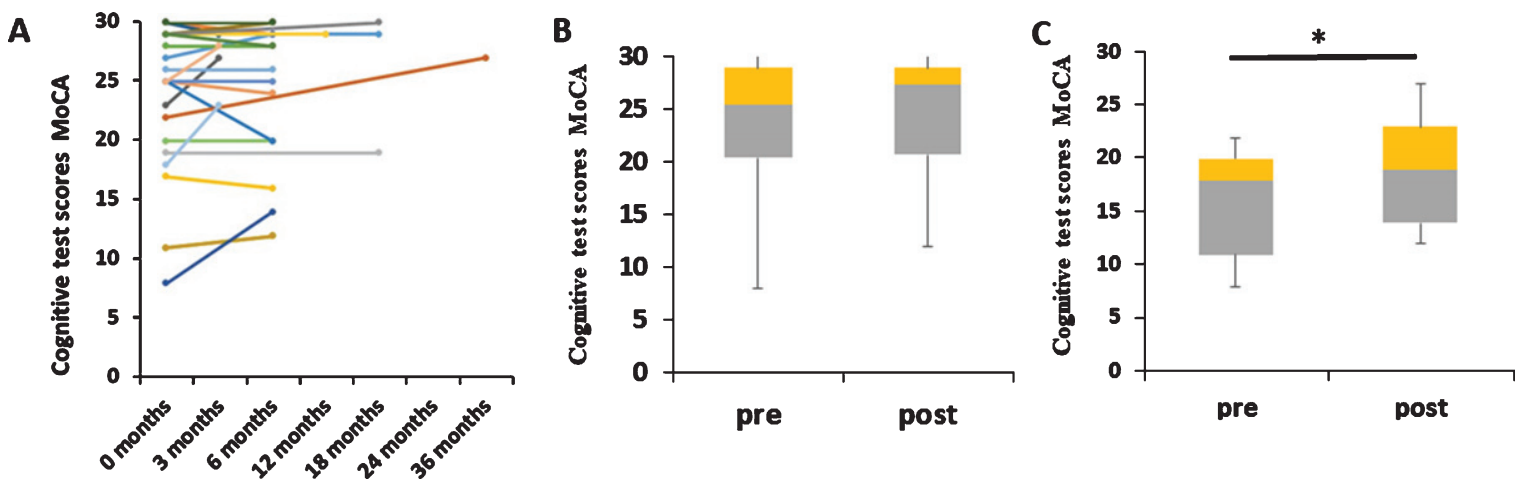

Fig. 1. Cognitive testing with the PTPr program. A) MoCA test scores from participants and baseline and indicated time points after inception of the program $(n=26)$. Out of 35, only 26 participants have both MoCA test scores, i.e., they have baseline and endpoint MoCA scores; therefore, only those participants were used to generate this figure. B) While there is a trend toward increased MoCA scores among the entire participant pool $(n=26)$, statistical significance was not achieved ( $p=0.121$, not significant). The last time point for a participant was used for statistical analysis. C) Among participants with MoCA scores below 24 at baseline, a statistically significant increase was observed $(n=9, p=0.029)$.

Table 2

Participants in the study

\begin{tabular}{lccccc}
\hline & \multicolumn{2}{c}{ Male } & & \multicolumn{2}{c}{ Female } \\
\cline { 2 - 3 } \cline { 5 - 6 } Demographic Characteristics & Actual & Percent & & Actual & Percent \\
\hline Total Participants $n=35$ & 13 & & & 22 & \\
Average Age & 75 & & & 69.72 & \\
APOE4 & 9 & $69.2 \%$ & & 17 & $73.9 \%$ \\
Average BMI & 23 & & & 20.2 & \\
Education: BA or Higher & 13 & $100.0 \%$ & & 17 & $81.8 \%$ \\
Baseline Cognitive Test & 3 & $23.0 \%$ & & 12 & $54.5 \%$ \\
$\quad$ SCI & 6 & $46.2 \%$ & & 3 & $13.6 \%$ \\
MCI & 2 & $15.3 \%$ & & 2 & $8.7 \%$ \\
AD & 2 & $15.3 \%$ & & 5 & $22.7 \%$ \\
No Score & & &
\end{tabular}

APOE4, apolipoprotein E4; BMI, body mass index; SCI, subjective cognitive impairment; MCI, mild cognitive impairment; $\mathrm{AD}$, Alzheimer's disease.

Table 2. In general, participants have a high education, are not overweight, and often have at least one APOE4 allele, a major risk factor for $\mathrm{AD}$ development.

A primary goal of the PTPr is to help people maintain or even improve cognitive performance after experiencing subjective cognitive impairment (SCI), mild cognitive impairment (MCI) or early stage AD. MoCA scores were collected at baseline and at various points after participation in the program. Cognitive performance steadily declines in MCI and AD patients. Our hypothesis was that this could be prevented or possibly reversed through a strategy to mitigate risk factors.

Follow up cognitive tests were taken at times ranging from 3 to 18 months, although most data points were at 3-6 months post participation in the pro-
Table 3

Participants and statistical $p$-values for each data analysis

\begin{tabular}{lcc}
\hline Figure & No of participants & $p$-value \\
\hline 1A & 26 & NA \\
1B & 26 & 0.121 \\
1C & 9 & 0.029 \\
2A & 35 & 0.181 \\
2B & 20 & 0.015 \\
2C & 34 & 0.109 \\
2D & 23 & 0.0003 \\
2E & 20 & 0.000014 \\
2F & 16 & 0.012 \\
2G & 10 & 0.013 \\
\hline
\end{tabular}

gram (Fig. 1A). To generate statistical relevance, we utilized the latest data point for the analysis. While there was an improvement in MoCA test score in the follow-up tests, data failed to reach statistical significance $(p=0.12)$ (Fig. 1B. See Table 3 for number of participants in each sub-figure and $p$-value calculations). No differences were observed between men and women. One limitation to reaching statistical significance is that many of the participants had high MoCA scores at the inception of the treatment, in part because participation was open to people with SCI. To assess changes in participants with lower MoCA scores at inception, we re-analyzed the data in people with scores less than 24. In this case significant improvement was observed $(p=0.03)$ (Fig. 1C). While participant size needs to be increased and additional tests including placebo-controlled studies need to be performed, these findings show promise that the PTPr can delay or even reverse cognitive performance. 
A

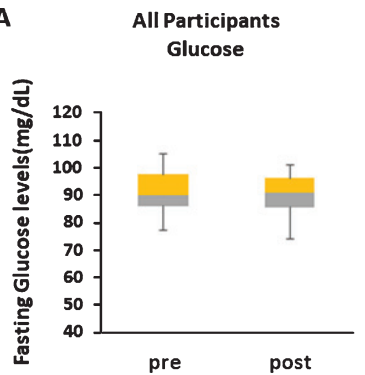

B

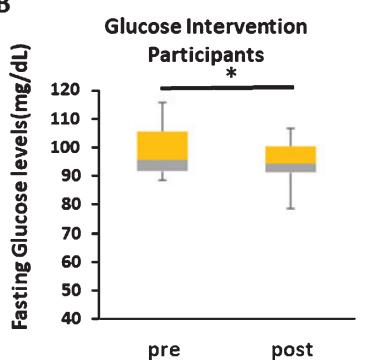

C

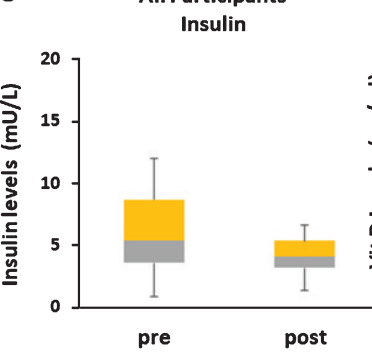

D

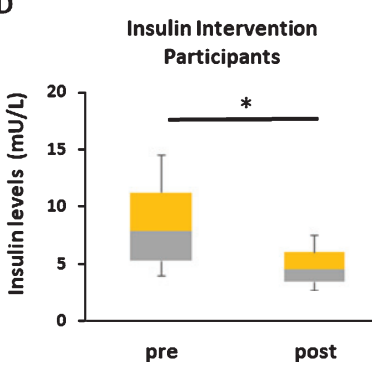

E

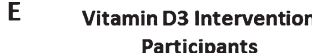

$*$

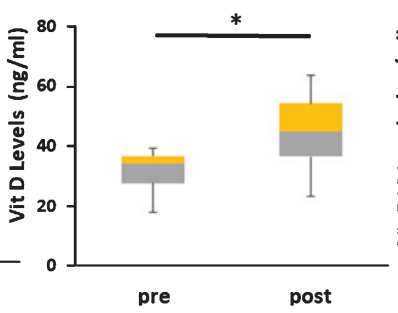

F

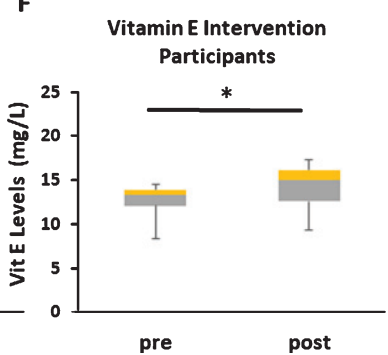

G

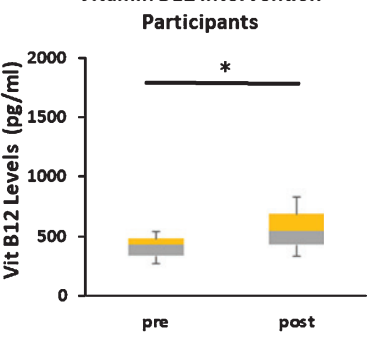

Fig. 2. Changes in risk factor levels among participants. A) No changes in blood glucose levels among the entire participant pool, but (B) a statistically significant reduction among participants with elevated blood glucose levels at inception. C) No significant changes in blood insulin levels among the entire participant pool, but (D) a statistically significant reduction among participants with elevated blood glucose levels at inception. Increased vitamin D3 (E), E (F), and B12 (G) levels among participants that had low levels at the inception of the program.

The PTPr seeks to offset a range of risk factors linked to $\mathrm{AD}$, making it possible to test whether these parameters, which are often linked to other chronic diseases of aging, can be altered. Many are metabolic in nature and linked to diseases including type II diabetes, cardiovascular disease and other conditions [23-26]. Therefore, we assessed whether individual parameters were improved through the educational program and interventions. Among our findings, we observe a significant decline in fasting blood glucose levels in participants where levels were high at baseline ( $>89 \mathrm{mg} / \mathrm{dL} ; p=0.02$ ) (Fig. 2A, B). Generally high fasting glucose is defined as greater than $100 \mathrm{mg} / \mathrm{dl}$; however, we chose a more stringent criteria based on previous studies [27]. In participants over $89 \mathrm{mg} / \mathrm{dL}$, education on diet and exercise was recommended, along with ginger and cinnamon [28-31]. In participants over $125 \mathrm{mg} / \mathrm{dL}, 500 \mathrm{mg}$ berberine was recommended twice a day with meals, followed by monitoring at 8 -week intervals [32]. Individuals with high fasting insulin levels (defined as levels above $4 \mathrm{mU} / \mathrm{L}$ ) also displayed significant improvements after adopting a similar set of recommendations for those who were hyperglycemic (Fig. 2C, D). Similarly, hemoglobin A1C and HOMA-IR levels were also reduced in participants with baseline scores above $5.2 \%$ (not shown). These findings indicate that participants in the PTPr experienced improved metabolic parameters, and therefore may be protected from other age-related conditions.

Among other risk factors that were effectively mitigated by the PTPr, we found that vitamins D3, E, and B12 were statistically increased in participants judged to have low levels at baseline (Fig. 2E-G). Free testosterone levels were also increased in males with baseline levels below $500 \mathrm{ng} / \mathrm{dL}$ (not shown). Total testosterone levels were not altered. Increases in zinc were also observed through direct supplementation in participants with low levels at baseline (not shown). Other parameters did not demonstrate statistically significant impact by the PTPr including cholesterol and inflammatory markers. This suggests that improvements in program strategies to effectively mitigate these and other risk factors may result in greater effects on cognitive performance.

\section{DISCUSSION}

$\mathrm{AD}$ affects over five million Americans. A chronic and progressive disease, recent estimates list $\mathrm{AD}$ as the third leading cause of death in the United States. In the absence of effective prevention and treatment, the prospects for the future are of great concern. With 13 million Americans and 160 million citizens globally 
projected to have $\mathrm{AD}$ by 2050, this raises concerns for the impact on healthcare systems worldwide, including the possible bankruptcy of Medicare. In the past decade alone, hundreds of clinical trials have been conducted for $\mathrm{AD}$ at an aggregate cost of billions of dollars, without significant success [1,2]. Extensive resources have been directed toward drug trials and this has led some to question whether the approach taken to focus almost exclusively on drug development for AD and its predecessors, SCI and MCI, is an optimal one. Ultimately, effective drugs may be developed, but other approaches such as treating risk factors may reduce disease onset and progression in the interim.

Chronic disease increases over the life course [33]. We suggest that a diverse approach is needed to target not only the known cause of the disease but also those factors that contribute to the progression and severity of the disease: lifestyle choices, stress, sleep, nutrient deficiencies, mental health, socialization, inflammation, and diet [22]. While research has focused on treating a single cause of $\mathrm{AD}$, to date there is no medical cure or single effective treatment, suggesting that there is more than one cause and solution. Increasing evidence supports dietary and other lifestyle changes as the most effective way to prevent, slow, or reverse disease progression. Studies also demonstrate that a more personalized and precision approach is needed for each individual participant to affect the greatest change and improvement.

To date, there are no single interventions that have resulted in stabilization or reversal of AD symptoms or progression. Although there are numerous studies looking at single interventions, including exercise, vitamin deficiencies, and glucose metabolism, they have not reported lasting effects for those suffering from a disease, which is largely deemed as progressive and irreversible.

Genetic evaluation for $\mathrm{AD}$ often includes testing for the $A P O \mathrm{E}$ gene, which is the strongest known predictor of AD [34]. The APOE4 gene has been linked to chronic inflammation and chronic disease later in life, including AD [35]. However, even though there may be a genetic predisposition for $\mathrm{AD}$, there is good evidence that lifestyle factors play a strong role in maintaining cognitive health as we age.

After a thorough review of published research, we have developed a comprehensive approach to addressing scientifically supported risk factors that have been rigorously defined as interventions to promote prevention, increased resiliency, and stabilization of brain function in the realm of $\mathrm{AD}$ and dementia. Utilizing cutting edge technology (PTP) in concert with in-person coaching and consultation, we are demonstrating that a multi-modal and personalized approach promotes an improved resiliency and restoration of optimal brain function.

The PTP includes genetics, an extensive blood panel, and lifestyle data to evaluate a number of metabolic factors and nutrient levels associated with cognitive health. Target laboratory levels for the PTP may be more stringent than standard laboratory ranges, as the goal is to reach optimized levels for cognitive health.

The earlier potential causes for cognitive decline are identified, the earlier steps can be taken to correct them and preserve cognitive health. Knowing this, our primary goal was to recruit participants that were in the early stages of disease (SCI, MCI, and early AD). The data for baseline MoCA score shows a range of 8-30 with the majority of patients scoring in the 20-30 range. Participants undergoing the program remained stable between baseline and follow-up. However, participants with a score of less than 24 showed a statistically significant improvement (see Fig. 1). This supports our conclusion that early intervention is critical, but also suggests that even with further disease progression, improvement can be observed. We also included a data point for a patient that had extended follow up over a 36-month period. This participant showed both short- and longterm progressive improvement over this time period, suggesting that the PTPr has both short and long- term effects. We included this long-term participant as an indicator of the potential benefits of the program, but of course more studies are necessary to establish its potential long-term benefits.

Our results clearly demonstrate that the metabolic impact of blood glucose metabolism is paramount to the improvement and stabilization of memory decline. All four parameters, fasting blood glucose, fasting insulin, hemoglobin A1c, and HOMA-insulin resistance scores, showed significant improvement with the PTPr (see Fig. 2). As AD has been recently labeled as 'type III diabetes', this is further support for the need for addressing blood glucose and its contribution to cognitive decline $[8,36]$.

We saw similar results with vitamin D3, vitamin E, vitamin B12, and zinc (see Fig. 2, not shown). Given that each of these alone has been studied in $\mathrm{AD}$ [37-39], but none have definitively shown to be linked to improvement or stabilization of cognition, our findings call for multi-modal approaches to treatment. As more data emerges, it may also be possible 
to make specific links between seemingly separate risk factors that are currently unknown.

An interesting outcome of the PTPr was that of the results of improved free testosterone [40]. Given the difficulties with testosterone replacement and the need for close monitoring and often specialization needed to administer this treatment safely and effectively, no participants were asked to pursue testosterone treatment. We presume that as a participant's metabolism, including their gastrointestinal system, begins to improve as the PTPr progressed, hormonal imbalances self-correct.

While our approach looks at more than 35 factors known to contribute to cognitive decline, our results demonstrate that certain of those factors are more affected than others. This indicates that there is a need to further develop our PTPr as more data and studies becomes available. This also suggests that we are moving toward a comprehensive, precision medicine approach to AD. Furthermore, this study supports the need for more effort to be directed toward an approach that focuses on a one size fits one, not one size fits all approach, and that comprehensively assesses all of the risk factors involved. In summary, our findings from this pilot study reinforce the potential benefits of a personalized approach targeting risk factors, and call for larger, placebo-controlled studies to provide further validation.

\section{ACKNOWLEDGMENTS}

This study was supported by Affirmativ Health, which is a digital therapeutics company that conducts research and delivers precision treatment plans to physicians and their patients living with Alzheimer's disease and cognitive disorders.

\section{CONFLICT OF INTEREST}

G.S., A.M., D.M.K., and B.K.K. are all affiliated with Affirmativ Health, for which D.M.K. also maintains an equity position.

\section{REFERENCES}

[1] Cummings J (2018) Lessons learned from Alzheimer disease: Clinical trials with negative outcomes. Clin Transl Sci 11, 147-152.

[2] Elmaleh DR, Farlow MR, Conti PS, Tompkins RG, Kundakovic L, Tanzi RE (2019) Developing effective Alzheimer's disease therapies: Clinical experience and future directions. J Alzheimers Dis 71, 715-732.
[3] Schneider LS, Mangialasche F, Andreasen N, Feldman H, Giacobini E, Jones R, Mantua V, Mecocci P, Pani L, Winblad B, Kivipelto M (2014) Clinical trials and late-stage drug development for Alzheimer's disease: An appraisal from 1984 to 2014. J Intern Med 275, 251-283.

[4] Castellani RJ, Plascencia-Villa G, Perry G (2019) The amyloid cascade and Alzheimer's disease therapeutics: Theory versus observation. Lab Invest 99, 958-970.

[5] Takeda S (2019) Tau propagation as a diagnostic and therapeutic target for dementia: Potentials and unanswered questions. Front Neurosci 13, 1274.

[6] Cline EN, Bicca MA, Viola KL, Klein WL (2018) The amyloid-beta oligomer hypothesis: Beginning of the third decade. J Alzheimers Dis 64, S567-S610.

[7] Frost GR, Jonas LA, Li YM (2019) Friend, foe or both? Immune activity in Alzheimer's disease. Front Aging Neurosci 11, 337.

[8] Kandimalla R, Thirumala V, Reddy PH (2017) Is Alzheimer's disease a type 3 diabetes? A critical appraisal. Biochim Biophys Acta Mol Basis Dis 1863, 1078-1089.

[9] Hane FT, Robinson M, Lee BY, Bai O, Leonenko Z, Albert MS (2017) Recent progress in Alzheimer's disease research, part 3: Diagnosis and treatment. J Alzheimers Dis 57, 645665 .

[10] Bhatti GK, Reddy AP, Reddy PH, Bhatti JS (2019) Lifestyle modifications and nutritional interventions in aging-associated cognitive decline and Alzheimer's disease. Front Aging Neurosci 11, 369.

[11] Crous-Bou M, Minguillon C, Gramunt N, Molinuevo JL (2017) Alzheimer's disease prevention: From risk factors to early intervention. Alzheimers Res Ther 9, 71.

[12] Silva MVF, Loures CMG, Alves LCV, de Souza LC, Borges KBG, Carvalho MDG (2019) Alzheimer's disease: Risk factors and potentially protective measures. J Biomed Sci $\mathbf{2 6}$, 33.

[13] Hickman RA, Faustin A, Wisniewski T (2016) Alzheimer disease and its growing epidemic: Risk factors, biomarkers, and the urgent need for therapeutics. Neurol Clin 34, 941953.

[14] Singh B, Parsaik AK, Mielke MM, Erwin PJ, Knopman DS, Petersen RC, Roberts RO (2014) Association of mediterranean diet with mild cognitive impairment and Alzheimer's disease: A systematic review and metaanalysis. J Alzheimers Dis 39, 271-282.

[15] Littlejohns TJ, Henley WE, Lang IA, Annweiler C, Beauchet O, Chaves PH, Fried L, Kestenbaum BR, Kuller LH, Langa KM, Lopez OL, Kos K, Soni M, Llewellyn DJ (2014) Vitamin D and the risk of dementia and Alzheimer disease. Neurology 83, 920-928.

[16] Caruso A, Nicoletti F, Gaetano A, Scaccianoce S (2019) Risk factors for Alzheimer's disease: Focus on stress. Front Pharmacol 10, 976.

[17] Ngandu T, Lehtisalo J, Solomon A, Levalahti E, Ahtiluoto S, Antikainen R, Backman L, Hanninen T, Jula A, Laatikainen T, Lindstrom J, Mangialasche F, Paajanen T, Pajala S, Peltonen M, Rauramaa R, Stigsdotter-Neely A, Strandberg T, Tuomilehto J, Soininen H, Kivipelto M (2015) A 2 year multidomain intervention of diet, exercise, cognitive training, and vascular risk monitoring versus control to prevent cognitive decline in at-risk elderly people (FINGER): A randomised controlled trial. Lancet 385, 2255-2263.

[18] Xu W, Tan L, Wang HF, Jiang T, Tan MS, Tan L, Zhao QF, Li JQ, Wang J, Yu JT (2015) Meta-analysis of modifiable risk factors for Alzheimer's disease. J Neurol Neurosurg Psychiatry 86, 1299-1306. 
[19] Mangialasche F, Kivipelto M, Solomon A, Fratiglioni L (2012) Dementia prevention: Current epidemiological evidence and future perspective. Alzheimers Res Ther 4, 6.

[20] Rosenberg A, Ngandu T, Rusanen M, Antikainen R, Backman L, Havulinna S, Hanninen T, Laatikainen T, Lehtisalo J, Levalahti E, Lindstrom J, Paajanen T, Peltonen M, Soininen H, Stigsdotter-Neely A, Strandberg T, Tuomilehto J, Solomon A, Kivipelto M (2018) Multidomain lifestyle intervention benefits a large elderly population at risk for cognitive decline and dementia regardless of baseline characteristics: The FINGER trial. Alzheimers Dement 14, 263-270.

[21] Bredesen DE, Amos EC, Canick J, Ackerley M, Raji C, Fiala M, Ahdidan J (2016) Reversal of cognitive decline in Alzheimer's disease. Aging (Albany NY) 8, 1250-1258.

[22] Bredesen DE (2014) Reversal of cognitive decline: A novel therapeutic program. Aging (Albany NY) 6, 707-717.

[23] Campos-Pena V, Toral-Rios D, Becerril-Perez F, SanchezTorres C, Delgado-Namorado Y, Torres-Ossorio E, FrancoBocanegra D, Carvajal K (2017) Metabolic syndrome as a risk factor for Alzheimer's disease: Is Abeta a crucial factor in both pathologies? Antioxid Redox Signal 26, 542-560.

[24] Camarda C, Pipia C, Azzarello D, Battaglini I, Romeo G, Chiodi M, Camarda R (2018) Vascular risk factors, vascular diseases, and imaging findings in a hospital-based cohort of mild cognitive impairment types. Curr Alzheimer Res 15, 679-690.

[25] Heijer T, Skoog I, Oudkerk M, de Leeuw FE, de Groot JC, Hofman A, Breteler MM (2003) Association between blood pressure levels over time and brain atrophy in the elderly. Neurobiol Aging 24, 307-313.

[26] van der Lee SJ, Teunissen CE, Pool R, Shipley MJ, Teumer A, Chouraki V, Melo van Lent D, Tynkkynen J, Fischer K, Hernesniemi J, Haller T, Singh-Manoux A, Verhoeven A, Willemsen G, de Leeuw FA, Wagner H, van Dongen J, Hertel J, Budde K, Willems van Dijk K, Weinhold L, Ikram MA, Pietzner M, Perola M, Wagner M, Friedrich N, Slagboom PE, Scheltens P, Yang Q, Gertzen RE, Egert S, Li S, Hankemeier T, van Beijsterveldt CEM, Vasan RS, Maier W, Peeters CFW, Jorgen Grabe H, Ramirez A, Seshadri S, Metspalu A, Kivimaki M, Salomaa V, Demirkan A, Boomsma DI, van der Flier WM, Amin N, van Duijn CM (2018) Circulating metabolites and general cognitive ability and dementia: Evidence from 11 cohort studies. Alzheimers Dement 14, 707-722.

[27] Shaye K, Amir T, Shlomo S, Yechezkel S (2012) Fasting glucose levels within the high normal range predict cardiovascular outcome. Am Heart J 164, 111-116.

[28] Li Y, Tran VH, Duke CC, Roufogalis BD (2012) Gingerols of Zingiber officinale enhance glucose uptake by increasing cell surface GLUT4 in cultured L6 myotubes. Planta Med 78, 1549-1555.
[29] Kirkham S, Akilen R, Sharma S, Tsiami A (2009) The potential of cinnamon to reduce blood glucose levels in patients with type 2 diabetes and insulin resistance. Diabetes Obes Metab 11, 1100-1113.

[30] Al-Amin ZM, Thomson M, Al-Qattan KK, PeltonenShalaby R, Ali M (2006) Anti-diabetic and hypolipidaemic properties of ginger (Zingiber officinale) in streptozotocininduced diabetic rats. Br J Nutr 96, 660-666.

[31] Im K, Issac A, Nm J, Ninan E, Maliakel B, Kuttan R (2014) Effects of the polyphenol content on the anti-diabetic activity of Cinnamomum zeylanicum extracts. Food Funct $\mathbf{5}$, 2208-2220.

[32] Dong H, Wang N, Zhao L, Lu F (2012) Berberine in the treatment of type 2 diabetes mellitus: A systemic review and meta-analysis. Evid Based Complement Alternat Med 2012, 591654.

[33] Kennedy BK, Berger SL, Brunet A, Campisi J, Cuervo AM, Epel ES, Franceschi C, Lithgow GJ, Morimoto RI, Pessin JE, Rando TA, Richardson A, Schadt EE, Wyss-Coray T, Sierra F (2014) Geroscience: Linking aging to chronic disease. Cell 159, 709-713.

[34] Yu JT, Tan L, Hardy J (2014) Apolipoprotein E in Alzheimer's disease: An update. Annu Rev Neurosci 37, 79-100.

[35] Uchoa MF, Moser VA, Pike CJ (2016) Interactions between inflammation, sex steroids, and Alzheimer's disease risk factors. Front Neuroendocrinol 43, 60-82.

[36] Leszek J, Trypka E, Tarasov VV, Ashraf GM, Aliev G (2017) Type 3 diabetes mellitus: A novel implication of Alzheimer's disease. Curr Top Med Chem 17, 1331-1335.

[37] Mullan K, Williams MA, Cardwell CR, McGuinness B, Passmore P, Silvestri G, Woodside JV, McKay GJ (2017) Serum concentrations of vitamin E and carotenoids are altered in Alzheimer's disease: A case-control study. Alzheimers Dement (N Y) 3, 432-439.

[38] Morris MS, Selhub J, Jacques PF (2012) Vitamin B-12 and folate status in relation to decline in scores on the minimental state examination in the framingham heart study. $J$ Am Geriatr Soc 60, 1457-1464.

[39] Moore EM, Ames D, Mander AG, Carne RP, Brodaty H, Woodward MC, Boundy K, Ellis KA, Bush AI, Faux NG, Martins RN, Masters CL, Rowe CC, Szoeke C, Watters DA (2014) Among vitamin B12 deficient older people, high folate levels are associated with worse cognitive function: Combined data from three cohorts. J Alzheimers Dis 39, 661-668.

[40] Barron AM, Pike CJ (2012) Sex hormones, aging, and Alzheimer's disease. Front Biosci (Elite Ed) 4, 976-997. 